Research Article

\title{
Numerical Investigation and Analysis of Intermediate Principal Stress Effects on Rock Failure Behaviors
}

\author{
Shuxin Deng $\mathbb{D}^{1},{ }^{1}$ Yonglai Zheng $\mathbb{D}^{2},{ }^{2}$ Cuizhou Yue $\mathbb{D}{ }^{2}$, and Le Van Tuan $\mathbb{D}^{3}$ \\ ${ }^{1}$ School of Mechanical Engineering, Nanjing University of Science and Technology, Nanjing 210094, China \\ ${ }^{2}$ College of Civil Engineering, Tongji University, Shanghai 200092, China \\ ${ }^{3}$ Department of Engineering and Technology in Constructions and Transportation, \\ VNU Hanoi University of Engineering and Technology, Address: 144 Xuan Thuy Street, Cau Giay District, Hanoi, Vietnam \\ Correspondence should be addressed to Shuxin Deng; dsx@njust.edu.cn
}

Received 21 August 2020; Revised 3 December 2020; Accepted 13 December 2020; Published 28 December 2020

Academic Editor: Xuzhen He

Copyright (c) 2020 Shuxin Deng et al. This is an open access article distributed under the Creative Commons Attribution License, which permits unrestricted use, distribution, and reproduction in any medium, provided the original work is properly cited.

\begin{abstract}
A series of numerical experiments have been conducted to investigate the intermediate principal stress effects on rock failure behaviors. The numerical results show that the strength and deformation of the rock samples are significantly affected by the intermediate principal stress. The effects are inconsistent in different intervals. As intermediate principal stress ratio $b$ increases, the rock strength increases initially and finally decreases. When $b$ is approximately equal to 0.5 , the strength of the rock sample reaches the maximum. In the microlevel, the intermediate principal stress affects the number and distribution of microcracks. The increase of the intermediate principal stress makes the projection of the microcracks on the loading plane change from uniform to uneven. On the one hand, the intermediate principal stress restricts the propagation of microcracks in the normal direction along the intermediate principal stress (or with a component in this direction), which will lead to an increase in the strength of the rock samples. On the other hand, the propagation of microcracks along the normal direction with small principal stress (or with a component in this direction) is promoted, which leads to a decrease in the strength of the rock sample. End friction can make the intermediate principal stress effect more significant because the friction of the loading end to the rock sample can result in stress deviation between the actual value and experimental value. Inhomogeneity of stress field induced by the change of stress states or end friction forces is the external factor of the intermediate principal stress effect. Also, the inhomogeneity of rock material itself is the internal factor. Intermediate principal stress will promote or restrict the failure of certain directions, thus affecting the overall strength of the rock samples. The numerical results can be very meaningful for stability analysis of rock masses in practical engineering.
\end{abstract}

\section{Introduction}

Natural rock masses are generally in a three-dimensional stress state, which can be defined by three mutually perpendicular stress components $\left(\sigma_{1}, \sigma_{2}\right.$, and $\left.\sigma_{3}\right)$. It is an important subject in rock mechanics to study rock failure behaviors under three-dimensional stress conditions. The effect of the intermediate principal stress on rocks has been studied for many years, but it is still an unsolved problem. The traditional considerations suggest that that shear failure will occur when the shear stress along some planes in the sample is too large [1]. The extreme values of shear stress in a material are related only to the largest and smallest principal stress (commonly denoted as $\sigma_{1}$ and $\sigma_{3}$ ). Based on such considerations, the influence of intermediate principal stress $\left(\sigma_{2}\right)$ on the experimental results is not taken into account. But three-dimensional unequal stress states are very common in engineering practice, and true triaxial rock strength measurements have shown that the intermediate principal stress does have an effect on rock strength. Many researchers [2-7] have conducted a larger number of true triaxial tests on different rock types such as Dunham dolomite, Solnhofen limestone, and granite, to investigate the behavior under triaxial stress conditions. They found that the strength increases firstly and then decreases with the increase of $\sigma_{2}$. 
At the same time, researchers have developed many numerical models or used commercial software to study the failure processes of rocks under polyaxial stress conditions. Cai [8] studied the influence of the intermediate principal stress on rock fracturing and strength near excavation boundaries using a FEM/DEM combined numerical tool. Shi and $\mathrm{Li}$ [9] conducted a true triaxial simulation of a homogeneous rock specimen using the Mohr-Coulomb perfect plastic model in FLAC3D software and found that the end friction can lead to an apparent effect of the intermediate principal stress, even though the rock has no intermediate principal stress effect. Tang and Hudson [10] have collected many simulation results using the RFPA code for studying the emergent properties of a heterogeneous microstructure. In these numerical studies, the phenomenon of the intermediate principal stress effect has been reproduced. However, the reason why the strength of some rocks firstly increases and then decreases with a continued increase in the intermediate principal stress is still unclear.

To provide a mechanism analysis from the microscopic level, a set of numerical experiments has been designed to conduct a detailed study on the intermediate principal stress effect on rock failure behaviors, including analysis of stresses, strains, failure model, acoustic emission (AE), crack developments, end frictions, and microuniformities. Based on the simulation results, the mechanism of intermediate principal stress effect has been discussed.

\section{Description of the Numerical Model}

2.1. Numerical Tool. In the recent studies [11-15], the discrete element method (DEM) has been widely used because it can overcome the continuity hypothesis of traditional continuum mechanics. The DEM was originally introduced by Cundall [16] for the analysis of rock mechanics and then introduced to granular materials by Cundall and Strack [17]. PFC (Particle Flow Code) software, developed based on the DEM, models materials composed of an assembly of variably-sized rigid particles that interact at contacts to represent both granular and solid materials. PFC models provide extensive flexibility in composing specimens of various packing arrangements and particle size distributions. Also, boundary conditions can be easily modified to accommodate a wide range of loading conditions [15]. PFC models are well suited to simulate the nonlinear behavior of large deformation and fracture in rock masses.

Particles in PFC models can be bonded to one another by using either contact bonds, parallel bonds, or both types of bonds $[14,18]$. As shown in Figure 1, the contact-bond glue is of a vanishingly small size that acts only at the contact point, while the parallel-bond glue is of a finite size that acts over a circular cross section lying between the particles [19]. The contact bond can only transmit a force, while the parallel bond can transmit both a force and a moment [20]. The parallel bonds are defined with five parameters, such as normal and shear bond strength, normal and shear bond stiffness, and the bond radius, among which the bond stiffness and bond radius are not assigned in the contactbond model. The contact or parallel bonds are broken if the

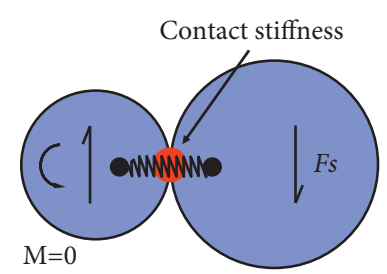

(a)

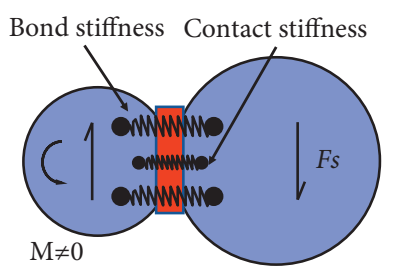

(b)
Figure 1: Bond models of PFC [18].

applied stresses are larger than the bond strengths [21, 22]. In the contact-bond model, bond breakage may not affect the macrostiffness significantly provided the particles remain in contact. However, in the parallel-bond model, bond breakage induces an immediate decrease in macrostiffness because the stiffness is contributed by both contact stiffness and bond stiffness [23]. Therefore, the parallel-bond model can be more suitable for rock material modeling in which the bonds may break in either tension or shearing with an associated reduction in stiffness [24]. For this reason, the parallel-bond model is selected in this study.

2.2. Numerical Model Generation. The effect of intermediate principal stress has been studied using PFC models consisting of spherical particles with radii between 2 and $3.23 \mathrm{~mm}$. As shown in Figure 2, all the spherical particles were randomly positioned inside a standard cuboid space $(50 \mathrm{~mm} \times 50 \mathrm{~mm} \times 100 \mathrm{~mm})$ comprised of four side plates (or walls), a bottom, and a loading plate. Microparameters of the model are calibrated with reference to the results of the uniaxial compression test of Beishan granites [25]. The calibration results are shown in Table 1, and a comparison between numerical and test results of granite is plotted in Figure 3, which indicates that the numerical model can well reflect the strength and deformation characteristics of Beishan granites.

2.3. Loading Method. In Figure 2, $X$-direction, $Y$-direction, and $Z$-direction correspond to the directions of major principal stress $\left(\sigma_{1}\right)$, intermediate principal stress $\left(\sigma_{2}\right)$, and minor principal stress $\left(\sigma_{3}\right)$, respectively. In three-dimensional unequal stress states, intermediate principal stress ratio $b$ is often used to present the magnitude of $\sigma_{2}$ relative to $\sigma_{1}$ and $\sigma_{3} . b$ is defined as

$$
b=\frac{\sigma_{2}-\sigma_{3}}{\sigma_{1}-\sigma_{3}} .
$$

When $\sigma_{3}$ was set to constant, the relationship $\Delta \sigma_{2}=$ $b \Delta \sigma_{1}$ could be derived from equation (1). For the simulated true triaxial tests, the top and button loading plates moved relatively with a specified velocity, which made $\sigma_{1}$ increase gradually. $\sigma_{2}$ was generated by the servosystem with the relationship $\Delta \sigma_{2}=b \Delta \sigma_{1}$. With the increase of axial stress $\left(\sigma_{1}\right)$, the specimen would be collapsed, and the failure behaviors were investigated. Different initial stress states were obtained by changing the values of $b$ and $\sigma_{3}$. 


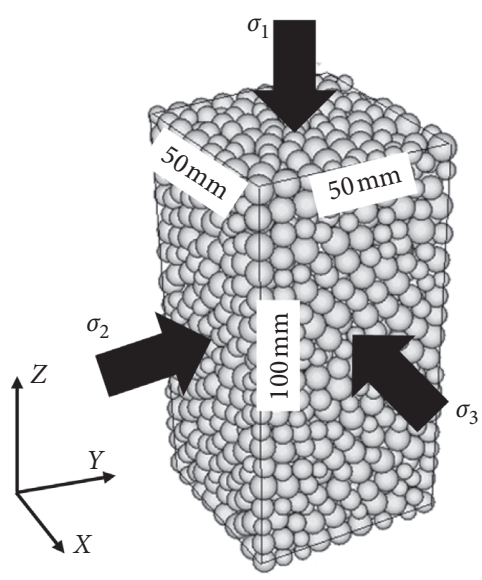

FIgure 2: Sample size and loading directions of the PFC model.

TABLe 1: Mesoscopic parameters of the PFC model.

\begin{tabular}{lc}
\hline Mesoscopic parameters & Values \\
\hline Ball-ball contact modulus $E_{c}(\mathrm{GPa})$ & 46.06 \\
Ball stiffness ratio $k_{n} / k_{s}$ & 1.15 \\
Ball friction coefficient $\mu$ & 0.25 \\
Parallel-bond modulus $\overline{E_{c}}(\mathrm{GPa})$ & 46.06 \\
Parallel-bond stiffness ratio $\overline{k_{n}} / \overline{k_{s}}$ & 1.15 \\
Parallel-bond normal strength, mean $\overline{\sigma_{c}}(\mathrm{MPa})$ & 178.11 \\
Parallel-bond shear strength, mean $\overline{\tau_{c}}(\mathrm{MPa})$ & 187.52 \\
Parallel-bond normal strength, std. dev. $(\mathrm{MPa})$ & 51.77 \\
Parallel-bond shear strength, std. dev. $(\mathrm{MPa})$ & 54.50 \\
\hline
\end{tabular}

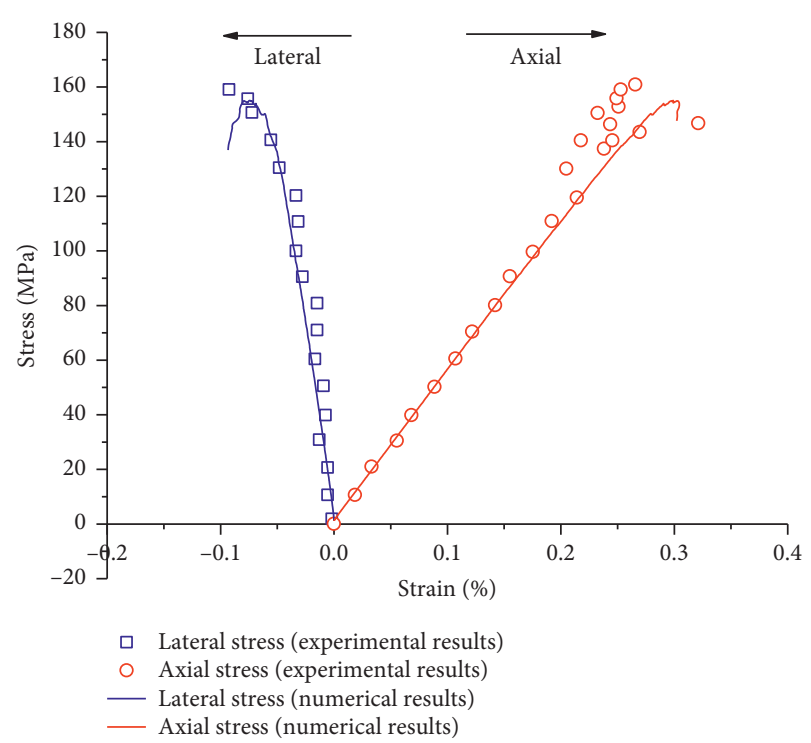

FIGURe 3: Comparison between numerical and experimental results of granite uniaxial compression.

\section{Analysis of Numerical Simulation Results}

3.1. Analysis of Stresses. Figure 4 shows the stress-strain curves of rock samples with different $b$ values in the whole failure process. During the loading process, $\sigma_{1}$ gradually

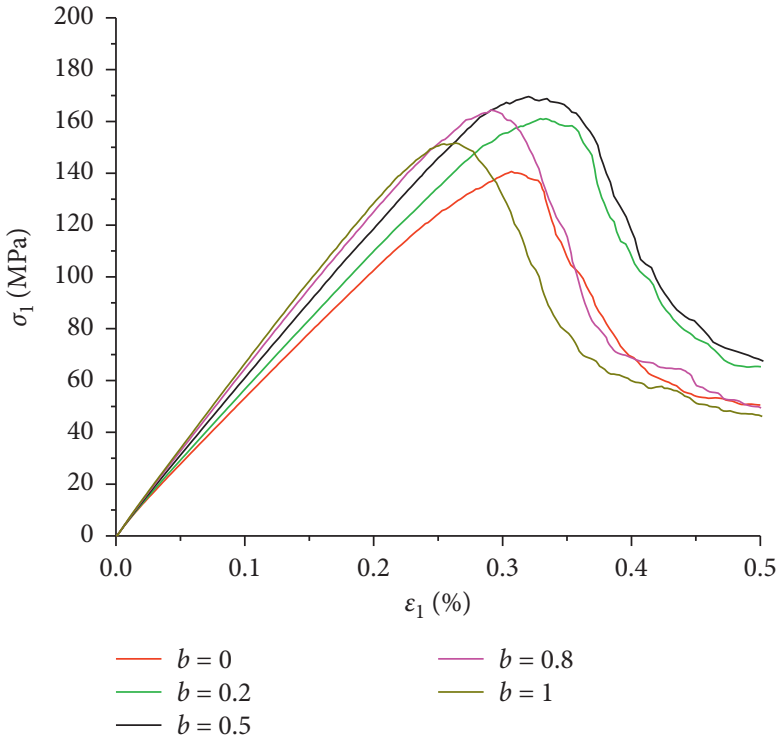

Figure 4: Stress-strain curves with different intermediate stress ratios.

increased and then decreased after reaching the peak. These stress-strain curves showed an obvious postpeak softening phenomenon, and the curve with $b=0.5$ had the maximum residual stress. In Figure 4, the amplitude of the stress reduction in the postpeak stage did not seem to depend on $b$. Before reaching the peak stress, with the increase of $b$, the elastic modulus increases, which means the intermediate principal stress can restrain the axial strain to some extent.

Peak stress curves with various $b$ are plotted in Figure 5. The intermediate principal stress has an obvious effect on the peak stress. With the increase of $b$, the peak stress also increases when $b$ is smaller; after the peak stress reaches the maximum, it gradually decreases with the increase of $b$. Also, the peak stress at $b=1$ is slightly larger than that at $b=0$. When the value of $b$ is about 0.5 , the peak stress reaches the maximum value. Compared with the conventional triaxial conditions $(b=0)$, the rock strength can be increased by up to about $20 \%$ under true triaxial conditions $(b>0)$.

3.2. Analysis of Strains. Figure 6 shows deviator stress-strain curves in three directions with various $b$. In the abscissa, the positive value denotes the compressive strain and the negative value denotes the tensile strain. Figure 6 indicates that, with the increase of $b$, the strain $\varepsilon_{2}$ in the intermediate stress direction changes from tension to compression, and the compressive strain increases continuously. In the process of $\varepsilon_{2}$ changing from tension to compression, there is a specific $b$ that makes $\varepsilon_{2}$ neither tension nor compression, namely, the plane strain state. From Figure 6, the model is close to the plane strain state when $b$ is approximately equal to 0.2 .

Relationships between volumetric strain and axial strain with different $b$ are plotted in Figure 7 . As the loading progresses, the model changes from shrinkage to dilation. The $\varepsilon_{1}$ corresponding to the critical state initially increases with the increase of $b$ and then gradually decreases. 


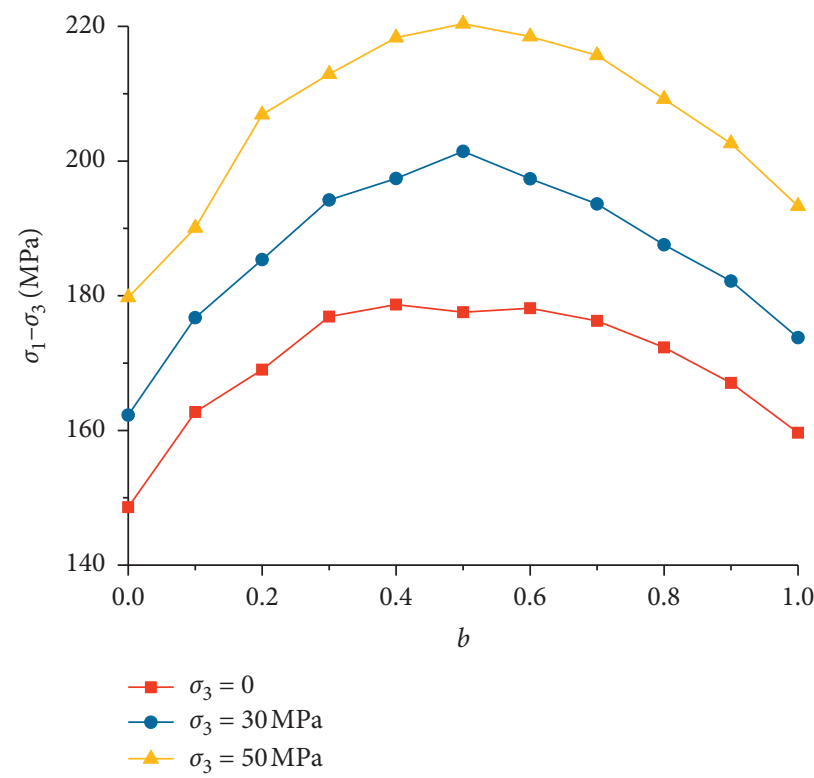

FIgURE 5: Peak stress curves with different intermediate stress ratios.

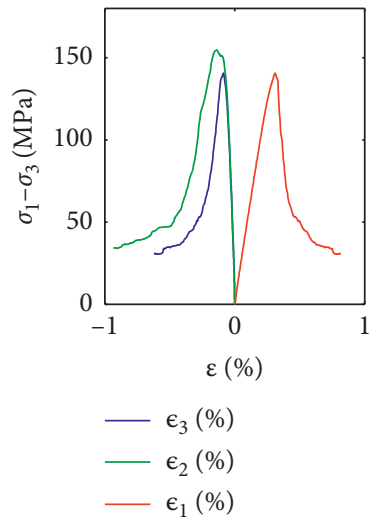

(a)

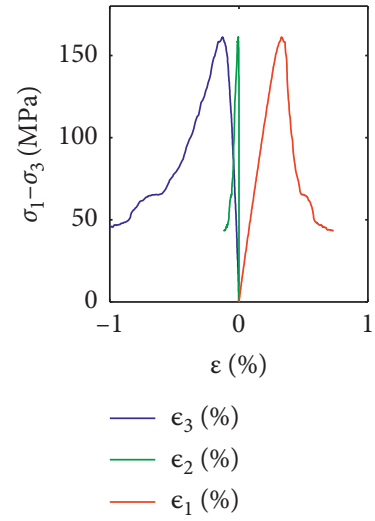

(b)

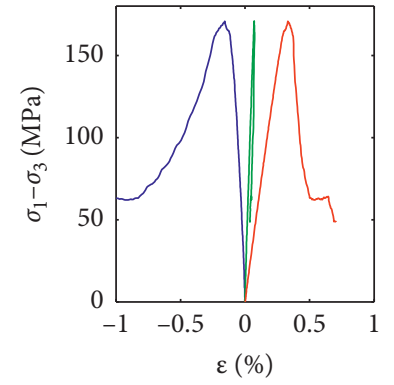

$$
\begin{aligned}
& -\epsilon_{3}(\%) \\
& -\epsilon_{2}(\%) \\
& -\epsilon_{1}(\%)
\end{aligned}
$$

(c)
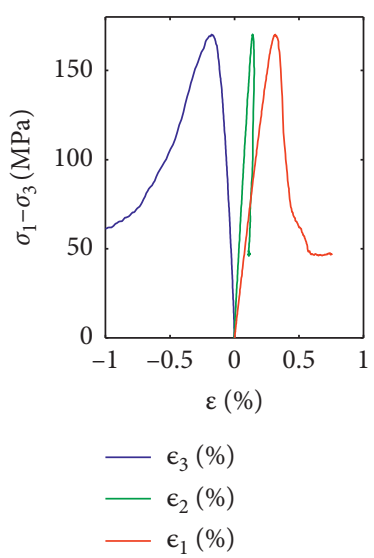

(d)

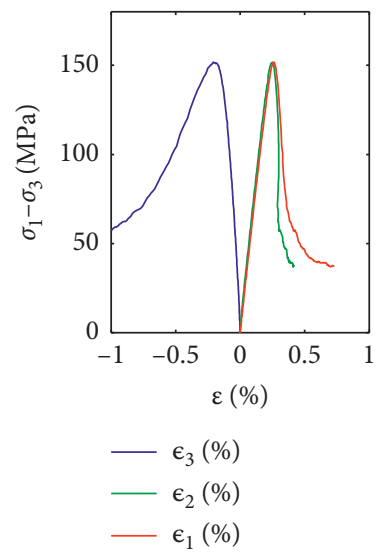

(e)

Figure 6: Deviator stress-strain curves in three directions. (a) $b=0$, (b) $b=0.2$, (c) $b=0.4$, (d) $b=0.8$, and (e) $b=1.0$. 


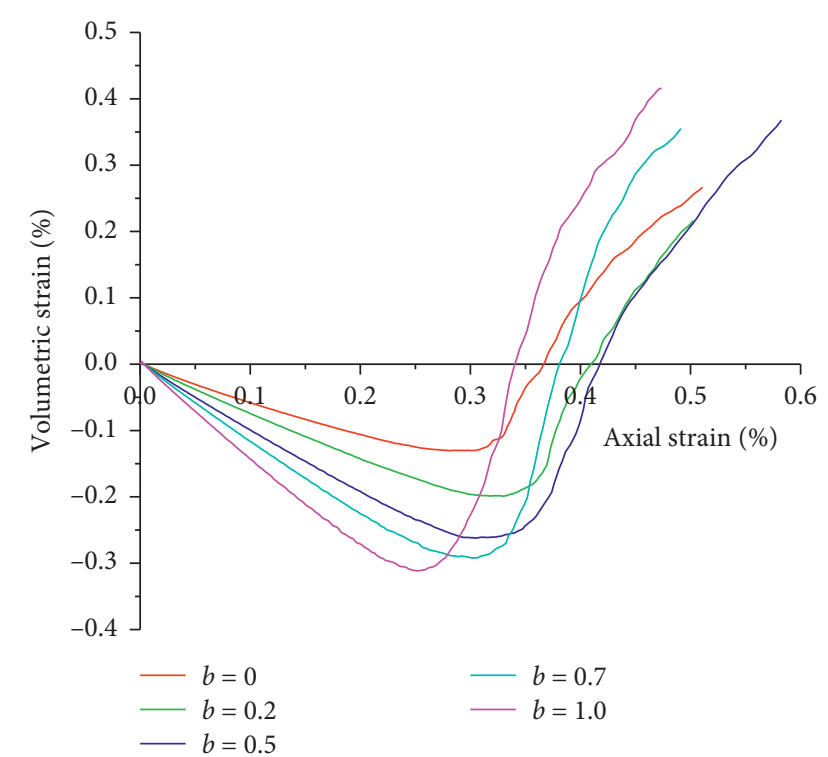

FIGURE 7: Relationships between volumetric strain and axial strain with different $b$.

3.3. Analysis of the Failure Model. Figure 8 shows the displacement contours of rock samples after failure under different intermediate principal stress levels. The failure mode of rock under three-dimensional stress conditions is shear slip. When $b$ is low, obvious slip surfaces are formed on both the $X Z$-plane and the $Y Z$-plane. When $b=0$, the slip surface distribution on the $X Z$-plane and $Y Z$-plane is almost symmetrical. As the value of $b$ increases, the slip surface on the YZ-plane gradually becomes inconspicuous until it disappears. It shows that the existence of the intermediate principal stress limits the initiation and propagation of microcracks parallel to the $X$-direction, which makes it difficult for the sample to form a slip surface on the $Y Z$ plane.

3.4. Analysis of Acoustic Emission (AE). Figure 9(a) presents the relationship between deviator stress, number of microcracks and $\mathrm{AE}$ events rate, and axial strain. Here, the formation of a microcrack represents an acoustic emission event [26-28], and the AE event rate in Figure 9(a) refers to the number of acoustic emission events for each $0.0174 \%$ axial strain.

In the initial elastic deformation stage, almost no microcracks are formed. As the loading progresses, microcracks gradually initiate. It can be seen from Figure 9(a) that the number of microcracks will increase rapidly as the sample approaches failure, and the AE event rate will have a maximum value and then gradually decreases. In the failure stage, a large number of microcracks in the sample suddenly sprung up and expanded rapidly, and the failure surface of the rock sample gradually formed at this stage.

Figures 9(b)-9(d) show the relationships between the AE event rate and the axial strain with different $b$. When $b$ is low, the rapid increase of microcracks in the sample showed a gradual delay, which means that, to generate the same amount of microcracks, it needs to be loaded for a longer time, with greater strain and axial stress. This leads to an increase in the strength of the rock sample. When $b$ is large, the time of rapid increase of microcracks tends to be gradually advanced. In addition, before the rapid increase in the number of cracks in the sample, the number of microcracks formed inside the sample will be larger than when $b$ is low. This will result in a decrease in the strength of the rock sample.

3.5. Analysis of Microcracks. From Section 3.3, it can be found that intermediate stress can affect the distribution of microcracks. For further research, we take a unit length in the normal direction for each crack, and the projection length in three directions is calculated. The projection length represents an equivalent number of microcracks with components in the direction, which can somehow characterize the failure effect in this direction. The statistical results of microcracks are shown in Figure 10.

From Figure 10(a), the number of microcracks in the $X$ direction decreases with the increase of $b$, while in the $Y$ direction, it is just the opposite. This means the damaging effect caused by the deviatoric stress $\sigma_{2}-\sigma_{3}$ will become significant as $b$ increases, and the enhancement of the damaging effect will have a weakening effect on the bearing capacity of the rock sample.

From Figure 10(b), it can be seen that the number of cracks distributed along the $Z$-direction on the $X Z$-plane is far larger than the number of microcracks distributed along the $X$-direction. Because $\sigma_{1}$ is always larger than $\sigma_{3}$, the $X\left(\sigma_{3}\right)$-direction is easier to form local tensile stress, which makes microcracks more conducive to initiation and expansion along the $Z$-direction. As $b$ increases, the number of microcracks in the $Z$-direction increases slightly, and the number of microcracks in the $X$-direction decreases slightly.

Figure 10 (c) shows that when $b<0.5$, the number of microcracks distributed along the $Z$-direction on the $Y Z$ plane decreases with the increase of $b$, and the number of microcracks along the $Y$-direction changes in an opposite way. Two curves intersect near $b=0.5$. When $b>0.5$, the number of microcracks along the $Z$-direction does not change as $b$ increases, and the growth rate of the number of microcracks along the $Y$-direction curve slows down. This means that the damaging effect caused by deviator stress $\sigma_{1}-\sigma_{2}$ will weaken as $b$ increases and then lead to an enhancement effect on rock strength. The enhanced effect in $b<0.5$ interval is obvious, but not obvious in $b>0.5$ interval.

3.6. Analysis of End Friction. In a physical experiment, there are contact friction forces between the sample and the pad, which will affect the internal force distribution of the rock sample.

To investigate the effect of the end friction, the coefficient of friction between the wall and the rock sample is set to 0 , $0.05,0.10,0.15$, and 0.20 . Figure 11 shows the curve of axial stress peaks with $b$ at a different coefficient of friction. Under the same stress state, as the friction coefficient increases, the axial stress peak increases, i.e., increasing the strength of the 


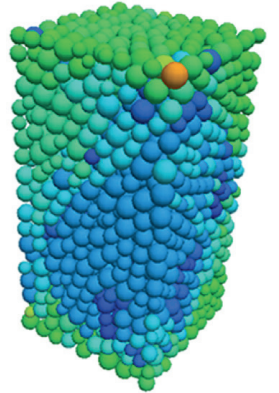

(a)

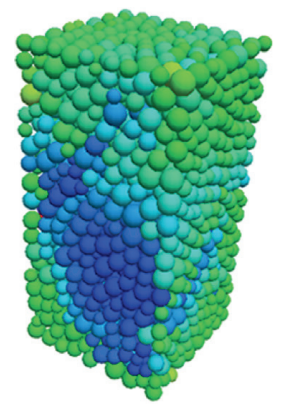

(e)

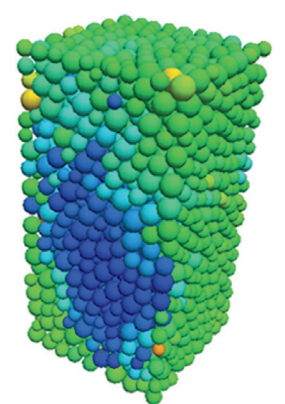

(i)

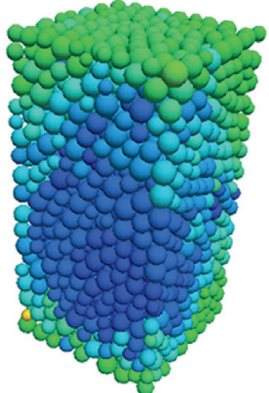

(b)

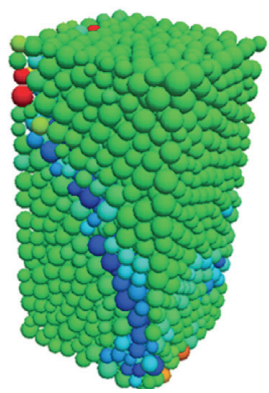

(f)

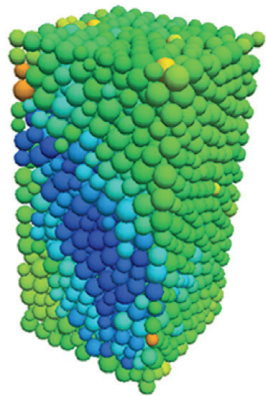

(j)

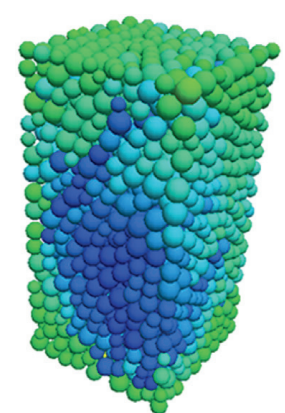

(c)

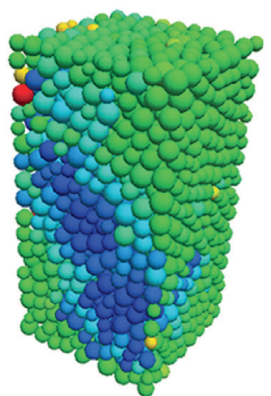

(g)

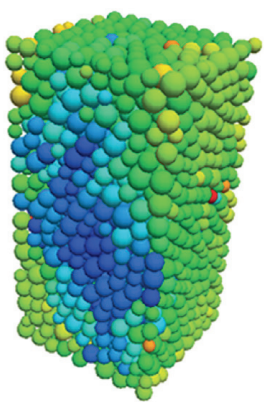

(k)

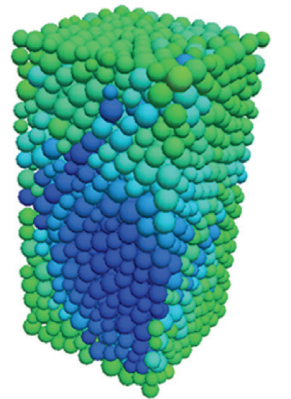

(d)

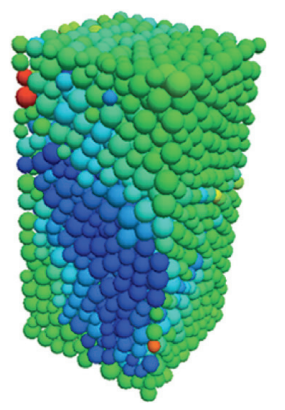

(h)

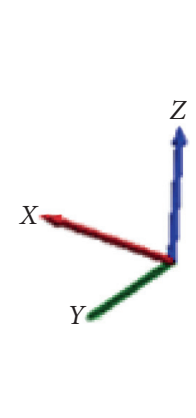

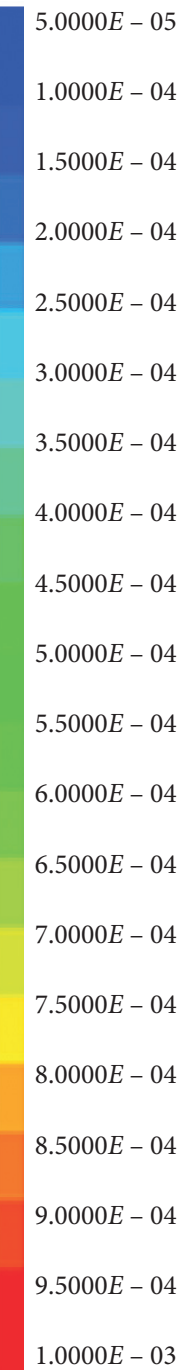

$1.0000 E-03$

Figure 8: Displacement contours of PFC models. (a) $b=0$, (b) $b=0.1$, (c) $b=0.2$, (d) $b=0.3$, (e) $b=0.4$, (f) $b=0.5$, (g) $b=0.6$, (h) $b=0.7$, (i) $b=0.8,(\mathrm{j}) b=0.9$, and $(\mathrm{k}) b=1$.

rock sample. When the coefficient of friction is $0,0.05,0.10$, 0.15 , and 0.20 , the rock strength can be increased by up to $20.23 \%, 23.07 \%, 25.60 \%, 25.48 \%$, and $26.43 \%$. As the friction coefficient increases, the intermediate principal stress effect on rock strength becomes more obvious. Due to the relative displacement of the wall and the rock sample, the rock sample is subjected to frictional force opposite to the displacement direction at the contact with the loading wall.

Since the rock sample is compressed along the axial direction, the friction force of the wall around the sample against the sample will generate tensile stress in the $\sigma_{1}$-direction inside the rock sample, which will offset part of the compressive stress generated by the axial load. Therefore, the measured value of $\sigma_{1}$ is greater than the actual value inside the sample. Similarly, in the $\sigma_{2}$ - and $\sigma_{3}$-direction, there is also a deviation between the measured value and the actual value. It can be seen from Figure 6 that, along the $\sigma_{3}$-direction, the measured value of $\sigma_{3}$ is smaller than the actual value as the sample is in a tensile state. Along the $\sigma_{2}$-direction, when $b$ is large, $\varepsilon_{2}$ appears to be compressed, and the measured value of $\varepsilon_{2}$ is greater than the actual value inside the sample.

3.7. Analysis of Microuniformity. The failure of rock material can be considered as the process of microcracks initiation and expansion, which is mainly due to the local tensile stress and shear stress generated inside the material. If the material is absolutely uniform, the deformation of the medium is coordinated under a complete compressive stress environment, and there will be no local tensile stress or shear stress in the medium before failure. Also, there will be no initiation of microcracks. Thus, the generation of microcracks is closely related to the uniformity of the material.

In Table 1, the coefficient of variation is approximately 0.33. To investigate the effect of microuniformity on the failure behavior of rock samples, the coefficient of variation is set to 0.4 and 0.5 . The average mesoscopic strength of the parallel bond remains the same. Comparison results of the stress peak are shown in Figure 12. Under the same stress 


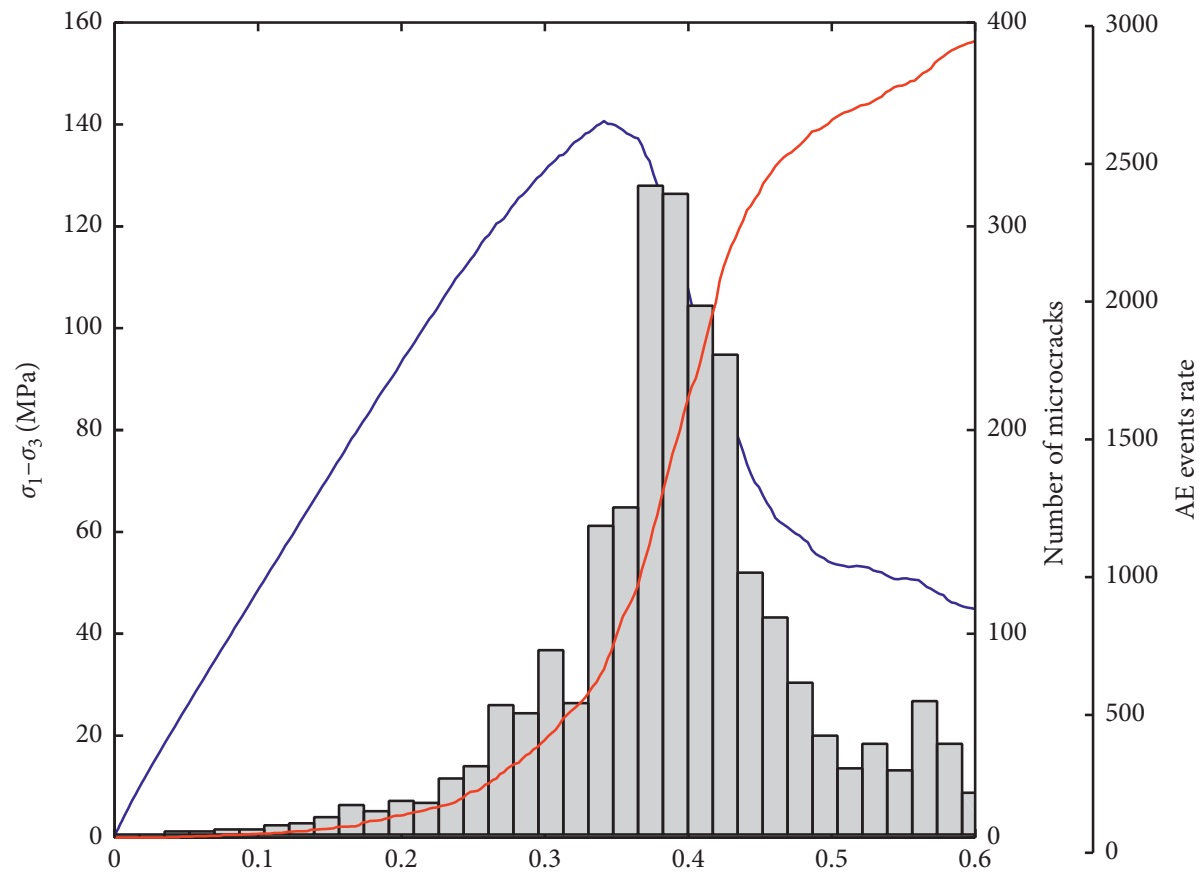

(a)

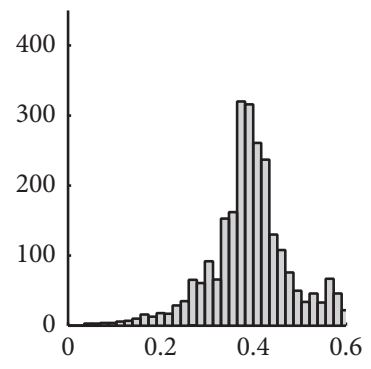

(b)

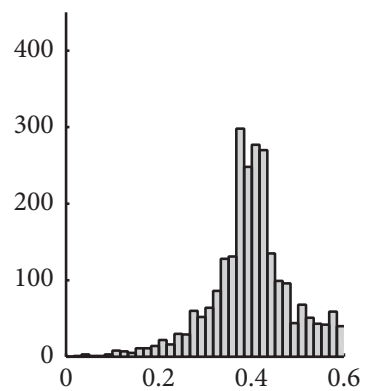

(e)

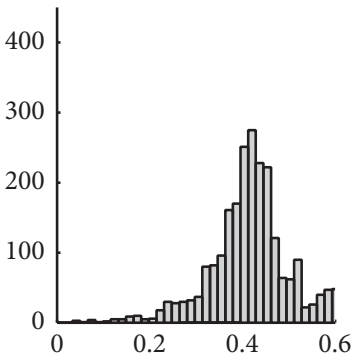

(c)

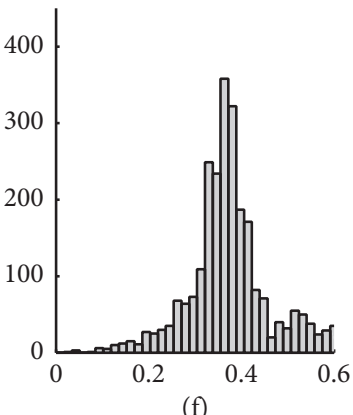

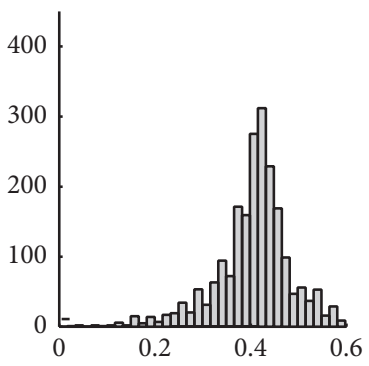

(d)

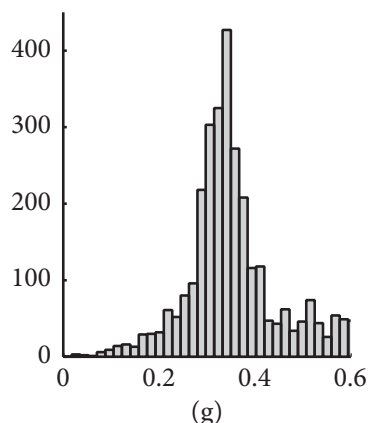

Figure 9: Acoustic emission events in different stress states. (a) $b=0$, (b) $b=0$, (c) $b=0.2$, (d) $b=0.4$, (e) $b=0.6$, (f) $b=0.8$, and (g) $b=1$.

state, the smaller the coefficient of variation, i.e., the more uniform the material, the greater the sample strength. According to the Weakest-Link Principle, a chain is only as strong as the weakest link.

When the average value of the bond strength is certain, the material with a smaller coefficient of variation can have a larger minimum value of the internal bond strength. That is, the strength of the weakest link is larger, which leads to an increase of the sample strength. Corresponding to the variation coefficient $0.33,0.40$, and 0.50 , the maximum value of the peak axial stress increases by $20.23 \%, 22.06 \%$, and
$30.83 \%$, respectively, compared to the stress state with $b=0$. That means for heterogeneous rock samples, the intermediate principal stress effect becomes more significant.

\section{Discussions}

From the abovementioned analysis of numerical simulation results, it can be seen that intermediate principal stress obviously affects the failure behavior of rock materials, and the effect is inconsistent in different intervals. As $b$ increases, 


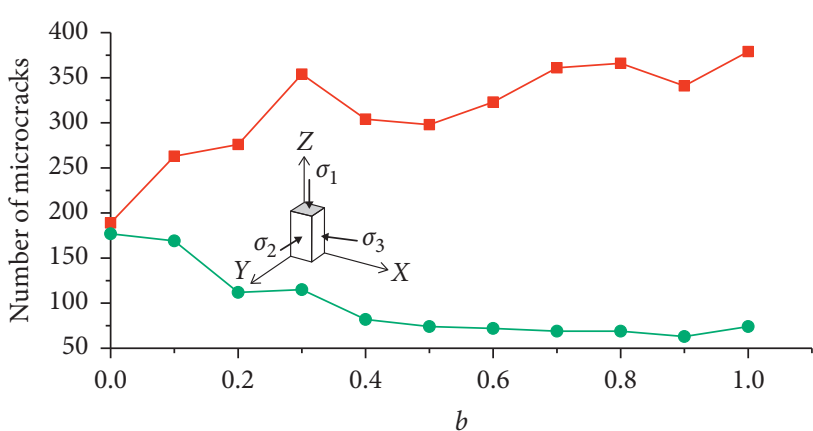

- - Along $X$-direction on $X Y$ plane - Along $Y$-direction on $X Y$ plane

(a)

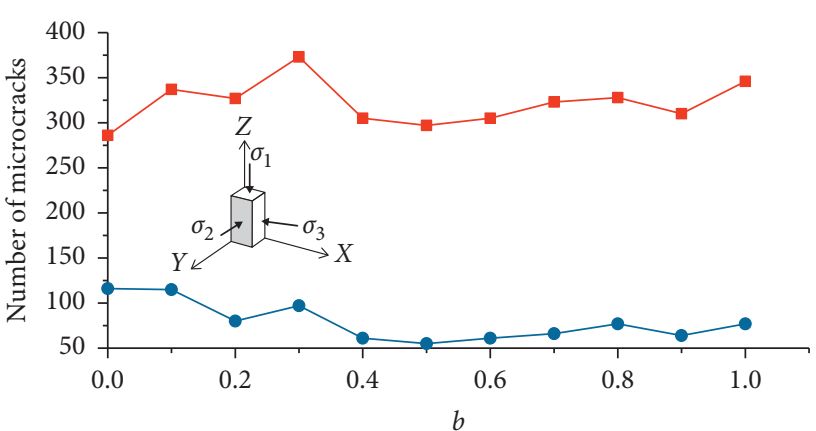

Along $X$-direction on $X Z$ plane Along $Z$-direction on $X Z$ plane

(b)

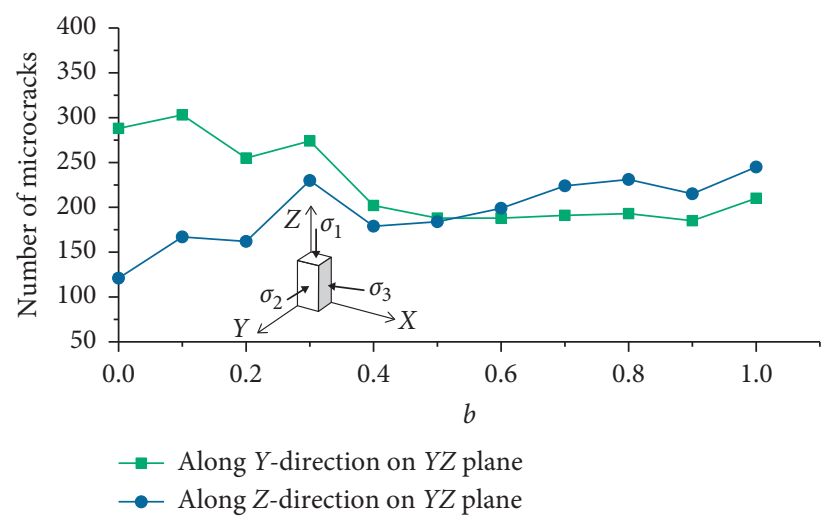

(c)

FIGURE 10: Distribution of microcracks in three directions.

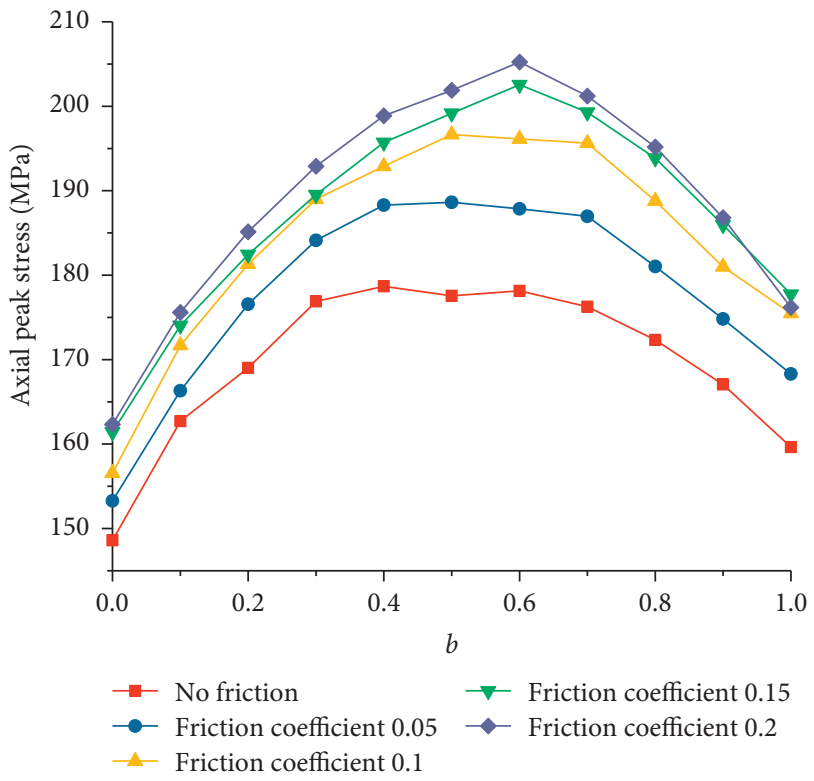

FIgURE 11: Effect of end friction on the axial peak stress.

the rock strength increases initially and finally decreases. When $b$ is approximately equal to 0.5 , the strength of the rock sample reaches the maximum.

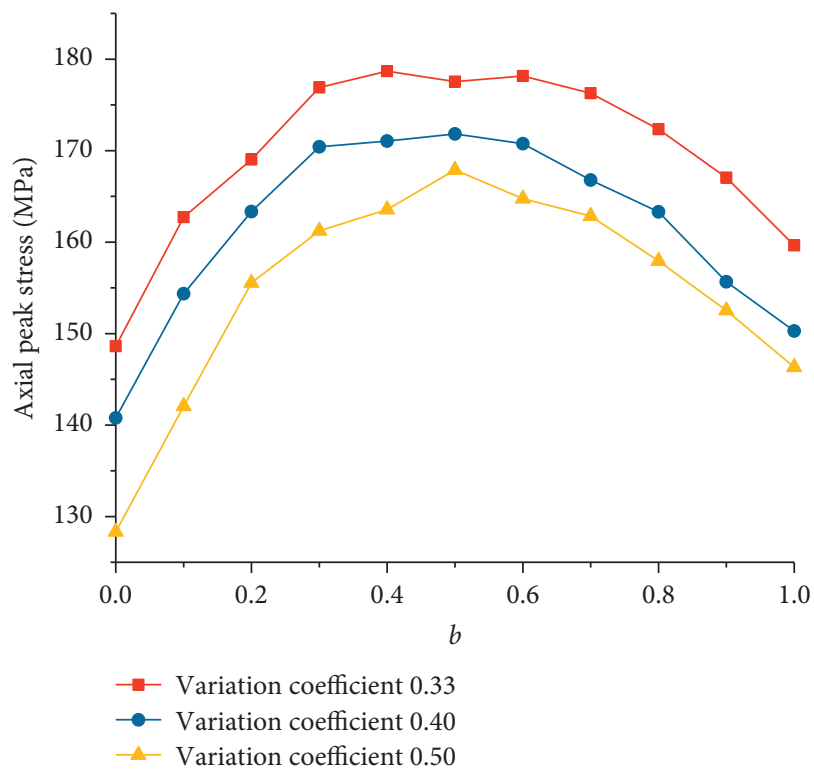

FIGURE 12: Effect of variation coefficient on the axial peak stress.

When $b=0$, namely, $\sigma_{2}=\sigma_{3}$, internal stresses are uniformly distributed. All directions of the microcracks constraint effect are equal, and microcracks will expand along the optimal direction. Since the generation of rock samples is 
completely random, the probability of microcracks expanding in all directions is the same, and the microcracks will be uniformly distributed (see Figure 10(a)). Slip surfaces occur on the $X Z$-plane and $Y Z$-plane (see Figure 8).

In the interval of $0<b<0.5$, as $\sigma_{2}>\sigma_{3}$, it is more difficult to produce local tensile stress in the $\sigma_{2}$ direction, thereby limiting initiation and expansion of the microcracks perpendicular to (or with vertical components) the $\sigma_{2}$-direction. In the $\sigma_{2}-\sigma_{3}$-plane, the number of microcracks along the $\sigma_{3}$-direction will continue to decrease as $\sigma_{2}$ increases (Figure 10(a)). As the expansion along the $\sigma_{3}$-direction is restricted, the probability of microcracks expanding along the $\sigma_{2}$-direction will be greatly increased and the number of microcracks along the $\sigma_{2}$-direction increases (see Figure 10(a)), which also makes the distribution of microcracks in the $X Y$-plane asymmetric (see Figure 6). Due to the restriction of $\sigma_{2}$, the rock sample needs more strain to produce a certain number of microcracks to form a macroslip surface, which leads to an increase of the rock strength.

In the interval of $0.5<b<1$, before the macroscopic slip surface is formed, the number of microcracks increases significantly with the increase of $\sigma_{2}$, which is not significant when $b<0.5$. The reason for this phenomenon is that, with the increase, the shear failure will occur not only in the $\sigma_{1}-\sigma_{2^{-}}$and $\sigma_{1}-\sigma_{3}$-direction, but also in the $\sigma_{2}-\sigma_{3}$ directions with a sufficiently large deviator stress. The increase in the number of microcracks in the yield stage of rock samples leads to a sudden outbreak of the growth of the number of microcracks in advance of time. Thus, in the interval of $0.5<b<1$, the strength of the rock samples decreases.

End friction can make the intermediate principal stress effect more significant because the friction of the loading end to the rock sample can result in stress deviation between the actual value and experimental value. As a result, the unevenness of the stress field of the sample is enhanced. The intermediate principal stress effect generated by the end friction is caused by the experimental error and does not reflect the mechanical properties of the material. When carrying out physical experiments, to obtain more accurate experimental results, the end friction should be eliminated.

Inhomogeneity of stress field induced by the change of stress states or end friction forces is the external factor of intermediate principal stress effect. Also, the inhomogeneity of rock material itself is the internal factor. For absolutely uniform material, due to the same nature of the material in all directions, shear failure of the material will occur along the direction of the maximum shear stress, i.e., the $\sigma_{1}-\sigma_{3}$-direction. In this case, the presence of intermediate principal stress does not affect the failure behavior of the material. For nonuniform materials, the nature of each direction is not the same, and there is a possibility of shear failure in each direction. In this case, intermediate principal stress will promote or restrict the failure of certain directions, thus affecting the overall strength of the rock sample.

In underground mining, the excavation of a tunnel may lead to fracturing and failure of the rock masses due to high induced stresses. The intermediate principal stress in the underground tunnel is often equal to the in situ stress along the tunnel axis, and the stress along the radial direction is $\sigma_{3}$, which is equal to zero at the excavation boundary [29]. Obviously, the stress near the excavation boundary is in the interval of $0.5<b<1$. As discussed above, propagation of the microcracks along the $\sigma_{2}$-direction is promoted, which leads to the formation of macroscopic cracks on the plane perpendicular to $\sigma_{2}$, namely, the generation of surface parallel fractures. This is the micromechanism of spalling failure. Densely spaced "onion-skin" fractures or slabs can be regularly formed in hard rock mines and deep civil tunnels [8].

The failure behavior of rock masses under three-dimensional stress conditions is a fundamental issue in rock engineering, which can provide more information to better understand the mechanism of complex geological phenomena. The numerical results in the present study can be helpful in the stability analysis of shaft drilling, underground mining, and tunnel excavation.

\section{Conclusions}

In this study, a series of numerical experiments have been conducted to investigate the intermediate principal stress effects on rock failure behaviors. In the numerical model, simulation results of stress, strain, failure model, Acoustic Emission (AE), microcracks, end friction, and microuniformity have been analyzed. Based on the simulation results, the mechanism of intermediate principal stress effect has been discussed. The conclusions are as follows:

(1) In the true triaxial compression experiment, strength and deformation of the rock samples are significantly affected by the intermediate principal stress. With the increase of $b$, the peak stress of the rock sample increases first and then decreases, and when $b=1$, the peak stress value is slightly greater than when $b=0$. As $b$ increases, the strain in the direction of the principal stress gradually changes from tension to compression. As the loading progresses, the model changes from shrinkage to dilation. The shear dilatancy characteristics become more obvious with the increase of $b$. The axial strain corresponding to the model changing from shear shrinkage to dilatancy increases with the increase of the $b$ value and then gradually decreases.

(2) At the microlevel, the intermediate principal stress will affect the number and distribution of microcracks. The increase of the intermediate principal stress makes the projection of the microcracks on the loading plane change from uniform to uneven. When $b>0.5$, the microcracks in the rock sample during the yield stage increase significantly. On the one hand, the intermediate principal stress restricts the propagation of microcracks in the normal direction along the intermediate principal stress (or with a component in this direction), which will lead to an increase in the strength of the rock sample. On the other hand, the propagation of microcracks along the normal direction with small principal stress (or with a component in this direction) is 
promoted, which leads to a decrease in the strength of the rock sample. The restricting or promoting effect on the growth of microcracks in different directions causes the macroscopic strength of the rock sample to increase first and then decrease.

(3) End friction will cause the deviation between the experimental and actual stress, which makes the intermediate principal stress effect more significant. The end effect cannot reflect the mechanical properties of the rock materials and should be eliminated during physical experiments.

(4) The heterogeneity of the material properties is an internal factor in the generation of the intermediate principal stress effect. For nonuniform materials, the properties of each direction are not the same, and there is a possibility of shear failure in each direction. The presence of the intermediate principal stress will promote or restrict the failure of certain directions, thus affecting the overall strength of the rock sample. The stronger the unevenness of the material, the more obvious the intermediate principal stress effect.

\section{Data Availability}

The experimental data used to support the findings of this study are available from the corresponding author upon request.

\section{Conflicts of Interest}

The authors declare that they have no conflicts of interest.

\section{Acknowledgments}

The authors acknowledge the financial support of the $\mathrm{Na}$ tional Natural Science Foundation of China (Grant no. 51909120).

\section{References}

[1] C. Jaeger, Rock Mechanics and Engineering, Cambridge University Press, Cambridge, UK, 1979.

[2] C. Chang and B. Haimson, "True triaxial strength and deformability of the German continental deep drilling program (KTB) deep hole amphibolite," Journal of Geophysical Research: Solid Earth, vol. 105, no. B8, pp. 18999-19013, 2000.

[3] B. Haimson, "True triaxial stresses and the brittle fracture of rock," in Rock Damage and Fluid Transport, Part I, pp. 1101-1130, Springer, Berlin, Germany, 2006.

[4] B. C. Haimson and C. Chang, "True triaxial strength of the KTB amphibolite under borehole wall conditions and its use to estimate the maximum horizontal in situ stress," Journal of Geophysical Research: Solid Earth, vol. 107, no. B10, 2002.

[5] K. Mogi, "Fracture and flow of rocks under high triaxial compression," Journal of Geophysical Research, vol. 76, no. 5, pp. 1255-1269, 1971.

[6] M. Takahashi and H. Koide, "Effect of the intermediate principal stress on strength and deformation behavior of sedimentary rocks at the depth shallower than $2000 \mathrm{~m}$," in Proceedings of the ISRM International Symposium, International Society for Rock Mechanics, Pau, France, August 1989.
[7] M. You, "True-triaxial strength criteria for rock," International Journal of Rock Mechanics and Mining Sciences, vol. 46, no. 1, pp. 115-127, 2009.

[8] M. Cai, "Influence of intermediate principal stress on rock fracturing and strength near excavation boundaries-Insight from numerical modeling," International Journal of Rock Mechanics and Mining Sciences, vol. 45, no. 5, pp. 763-772, 2008.

[9] L. Shi and X. Li, "Analysis of end friction effect in true triaxial test," Rock and Soil Mechanics, vol. 4, p. 068, 2009.

[10] C. Tang and J. Hudson, Rock Failure Mechanisms, CRC Press, Boca Raton, FL, USA, 2010.

[11] P.-Z. Pan, X.-T. Feng, and J. A. Hudson, "The influence of the intermediate principal stress on rock failure behaviour: a numerical study," Engineering Geology, vol. 124, pp. 109-118, 2012.

[12] Y. Cheng and W. Yang, "Influence of microscopic parameters on the stress-strain relation in rocks," Advances in Civil Engineering, vol. 2018, Article ID 7050468, 7 pages, 2018

[13] Y. Tian, L. Wang, H. Jin et al., "Dynamic response of seismic dangerous rock based on PFC and dynamics," Advances in Civil Engineering, vol. 2020, Article ID 8846130, 19 pages, 2020.

[14] S.-R. Zhang, B. Sun, C. Wang, and L. Yan, "Influence of intermediate principal stress on failure mechanism of hard rock with a pre-existing circular opening," Journal of Central South University, vol. 21, no. 4, pp. 1571-1582, 2014.

[15] E. Fjaer and H. Ruistuen, "Impact of the intermediate principal stress on the strength of heterogeneous rock," Journal of Geophysical Research: Solid Earth, vol. 107, no. B2, 2002.

[16] P. A. Cundall, "A computer model for simulating progressive, large-scale movements in blocky rock systems," in Proceedings of the International Symposium on Society of Rock Mechanics, vol. 1, pp. 2-8, Nancy, france, October 1971.

[17] P. A. Cundall and O. D. L. Strack, "A discrete numerical model for granular assemblies," Géotechnique, vol. 29, no. 1, pp. 47-65, 1979.

[18] Q. Zhang, X. Wang, L. Tian et al., "Analysis of mechanical and acoustic emission characteristics of rock materials with double-hole defects based on particle flow code," Shock and Vibration, vol. 2018, Article ID 7065029, 11 pages, 2018.

[19] S. S. Hashemi, A. A. Momeni, and N. Melkoumian, "Investigation of borehole stability in poorly cemented granular formations by discrete element method," Journal of Petroleum Science and Engineering, vol. 113, pp. 23-35, 2014.

[20] H. Yu and S. Shen, "Impact of aggregate packing on dynamic modulus of hot mix asphalt mixtures using three-dimensional discrete element method," Construction and Building Materials, vol. 26, no. 1, pp. 302-309, 2012.

[21] D. O. Potyondy and P. A. Cundall, "A bonded-particle model for rock," International Journal of Rock Mechanics and Mining Sciences, vol. 41, no. 8, pp. 1329-1364, 2004.

[22] P. A. Cundall and O. D. L. Strack, Particle Flow Code in 2 Dimensions, Itasca Consulting Group, Inc., Minneapolis, MN, USA, 1999.

[23] A. Manouchehrian, M. Sharifzadeh, M. F. Marji, and J. Gholamnejad, "A bonded particle model for analysis of the flaw orientation effect on crack propagation mechanism in brittle materials under compression," Archives of Civil and Mechanical Engineering, vol. 14, no. 1, pp. 40-52, 2014.

[24] N. Cho, C. D. Martin, and D. C. Sego, "A clumped particle model for rock," International Journal of Rock Mechanics and Mining Sciences, vol. 44, no. 7, pp. 997-1010, 2007. 
[25] L. Chen, J. Liu, C. Wang et al., "Elastoplastic damage model of Beishan deep granite," Yanshilixue Yu Gongcheng Xuebao/ Chinese Journal of Rock Mechanics and Engineering, vol. 32, pp. 289-298, 2013.

[26] M. Cai, P. K. Kaiser, H. Morioka et al., "FLAC/PFC coupled numerical simulation of AE in large-scale underground excavations," International Journal of Rock Mechanics and Mining Sciences, vol. 44, no. 4, pp. 550-564, 2007.

[27] H. Konietzky, "Numerical modeling in micromechanics via particle methods," in Proceedings of the 2nd International PFC Symposium, Kyoto, Japan, October 2004.

[28] Y. Zhang, Z. Shao, W. Wei, and R. Qiao, "PFC simulation of crack evolution and energy conversion during basalt failure process," Journal of Geophysics and Engineering, vol. 16, no. 3, pp. 639-651, 2019.

[29] E. Hoek and E. T. Brown, Underground Excavations in Rock, CRC Press, Boca Raton, FL, USA, 1980. 